\title{
NON-LINEAR PHENOMENA IN SOCIO-ECONOMIC SYSTEMS WITH NATURAL RESOURCE MANAGEMENT
}

\author{
J. A. Amador ${ }^{1}$, H. A. Granada, G. Olivar
}

Departamento de Ingeniería Eléctrica, Electrónica y Computación, Universidad Nacional de Colombia, Sede Manizales Campus La Nubia, Manizales, Colombia.

1 jaamadorm@unal.edu.co

\begin{abstract}
The concept of sustainable development implies worldwide responsibility and shift to a more sustainable lifestyles and patterns of consumption and production to obtain the harmony among society, economy, and nature. Nowadays, sustainable development is extensively described and studied, but very few works are dedicated to mathematical modelling techniques and numerical simulations. This work deals with some systems of ordinary differential equations in order to explain each one of the sustainable development dimensions (resources, economy, and social growth) and their dependence with population growth. As a result non-linear phenomena such as bifurcations and chaotic behavior may appear and help to explain some sustainability conditions revealing new features of sustainable development dynamics. Non-smooth phenomena such as sliding may appear when discontinuities are introduced into the system turning them into Filippov systems.
\end{abstract}

Keywords: Sustainable Development, Non-linear phenomena, Filippov system.

\section{INTRODUCTION}

To achieve an increase in the food production there are three ways we can do it: increasing cropland, improving yield, and reducing losses since contributions from aquaculture and synthetic food are negligible with respect to farming. The increased yield in agriculture has been the main objective in Food Crisis trying to cover more efficiently the regions most in need. For this purpose, new varieties of seeds have been created allowing more than one harvest per year and resistance and tolerance to plants diseases, pests, irregular irrigation, and poor soils. These "benefits" have resulted in an increase of the population engaged in agriculture that seeks to improve their income to support themselves and their family. However, not all impacts are positive since the intensification of agriculture reduces the use of seed diversity by increasing the implementation of single crop farming, the use of agrochemicals, and deforestation in order to expand arable areas; which results in the medium to long term in soil depletion and the reduction of renewable and nonrenewable resources reducing the chances of future productions doing this kind of agriculture, unsustainable.

The current Indian tribes occupying regions with abundant natural resources are at risk of failing as Easter Island did or migrate to other lands as did the Mayan civilization because 
of overharvesting their resources. This risk usually occurs not because of the mismanagement that they give to their own natural resources but because of the mismanagement that foreigners have been giving to these resources in a process of colonization and unsustainable exploitation. Is the case of the Amazon rainforest where its native inhabitants have been living there for thousands of years and have found the perfect harmony with nature taking just enough to survive by avoiding over-exploitation and promoting generation after generation the conservative culture. Was the process of colonization who brought the problems to the environmental conservation. These foreigners brought with them not only the implementation of extensive agriculture and mining but also the subjugation of the natives to use these activities falling into a constant contribution to environmental damage.

This interaction between population and the exploitation of natural resources has been studied through sets of ordinary differential equations (ODEs) related to the Lotka-Volterra model, where population is the predator and renewable resources represent the prey. The main results of these studies were focused on studying how institutional reforms reflected in changes in parameter values could prevent the fall of historical civilizations such as Easter Island whose mismanagement of natural resources was the cause of failure [1-4]. The simple model developed by Brander and Taylor [1] give us an intuitive reasoning about the fall of the civilizaction on Easter Island which could be repeated in current societies such as those inhabiting the Amazon rainforest and many others around the world who survive mainly from natural resource. An extension of this model was developed by D'Alessandro [5]. He introduced agriculture as a second source of income in order to obtain a more general structure of the linkages between ecological and economic systems.

The purpose of this paper is to study the dynamic properties and nonlinear phenomena such as bifurcations that can occur in different sets of ODEs in an ideal society which is assumed to comply with the parameter values. Both systems will be studied where the population uses renewable resources as the only source of livelihood as well as systems where agriculture has been introduced as a second source of income.

For this purpose we used two different types of dynamic systems: the so-called continuous or smooth dynamical systems whose states evolve continuously over time and the so-called discontinuous whose states change abruptly. Discontinuous dynamical systems discussed in this document are Filippov system or commonly called piecewise-smooth systems [6]. Both continuous systems and Filippov systems are described by standard differential equations of the form

$$
\dot{x}=f(x(t), \mu), \quad x \in \mathbb{R}^{n},
$$

where $f$ may be continuous or discontinuous.

The paper is organized as follows: Section 1 presents and analyzes the dynamics of a simple model where the only source of livelihood is the use of renewable resources. Then, Section 2 introduces the agriculture as a second source of income by using the model proposed in [5] which has interesting nonlinear phenomena. Section 3 presents a model where technological progress has been introduced as a third differential equation showing chaotic behavior. Section 4 introduces two ways of mitigating environmental degradation: $i$ ) through cultural changes and ii) through strict policies that seek to preserve an amount of resources, in this 
case smooth systems become Filippov system. Finally Section 5 presents some concluding remarks.

\section{Simple model of renewable resources and population growth}

Economic activities in the primary sector ${ }^{1}$ as uncontrolled extraction of wood in forests and rainforests are against the environment since humans use these resources to produce income to survive and ignore the importance of conserving natural resources for future generations. This complex relationship can be represented by the combination of the population and renewable resources dynamics.

\subsection{Mathematical model}

According to Malthus [7], an increase in per-capita income leads to an increment in population size; but at the same time this augmentation reduces chances of improve income. To represent this behavior we can use a hybrid model of the theories of Ricardo [7] and Malthus [8] as D'Alessandro [5] did. In his model, population dynamics is written in term of calories consumed and the natural level of calories needed to survive. This means that population increases if the level of calories consumed is greater than the natural level. On the other hand the dynamic of renewable resources depends on their natural growth and the harvesting rate. The first model representing this interaction is

$$
\left\{\begin{array}{l}
\dot{S}=\left[\rho\left(\frac{S}{k}-1\right)\left(1-\frac{S}{K}\right)-\alpha \beta L\right] S, \\
\dot{L}=(\phi \alpha \beta S-\sigma) L .
\end{array}\right.
$$

Where $\dot{S}$ and $\dot{L}$ represent the change over time of resources and population respectively. An increase in resources stock, produces an increment in their reproductive ability ${ }^{2}$ resulting an accumulation of resources which are limited by their carrying capacity $K$. Parameter $k$ is the resource stock where the growth rate becomes negative and the renewable resource tends to the extinction. $\rho$ is an intrinsic parameter of the resources that represents their regeneration rate. $\alpha$ is a measure of the technology used in harvesting, $\beta$ indicates the percentage of population dedicated to harvesting. Finally, $\phi$ and $\sigma$ are the caloric value of the resource and the natural caloric level respectively.

For convenience we define $s=\frac{S}{K}$ as the available percentage of resources at any time $t$. This implies that $s \in[0,1]$ and the growth rate becomes negative when $s=\frac{k}{K}=0.0583$, i.e. below $\% 5.83$ of resources the extinction is unavoidable.

\subsection{Equilibrium points}

System (1) is an autonomous system whose equilibria can be calculated by equalling simultaneously to zero both equations, $f(x(t), \mu)=0$. This system has four equilibria, three

\footnotetext{
${ }^{1}$ The primary sector of the economy extracts or harvests products of the soil, and most of times these products are considered raw materials for industries in the secondary sector. Some activities associated with the primary sector include agriculture, mining, forestry, hunting, and fishing.

${ }^{2}$ For instance, according to plant succession theory more trees can host more insects and birds which are largely responsible of pollination.
} 
of which are trivial equilibria and one internal equilibrium whose stability depends strongly on parameter values. Equilibria are the following solutions $(L, S)$.

Trivial equilibria

$$
\begin{aligned}
& P 1=(0,0), \\
& P 2=(0, k), \\
& P 3=(0, K),
\end{aligned}
$$

internal equilibrium

$$
P 4=\left(-\frac{\rho\left(-\sigma K \phi \alpha \beta+\sigma^{2}+k K \phi^{2} \alpha^{2} \beta^{2}-k \sigma \phi \alpha \beta\right)}{k K \phi^{2} \alpha^{3} \beta^{3}}, \frac{\sigma}{\phi \alpha \beta}\right) .
$$

\subsection{Steady-state analysis}

Temporal evolution of a dynamic system brings together two behaviors, the transient and the steady state. For purpose of this work we focus the results on studying the steady state. This state is reached when the state variables $L$ and $S$ does not change over time.

Assuming parameter values reported in Table 1 and an initial condition $\left(L_{0}, s_{0}\right)=$ $(40,1)$ we can implement a Runge-Kutta method to find the numerical solution of system (1) since analytical solution is not available

Table 1. Parameter Values

\begin{tabular}{l|c}
\hline Parameter & Value \\
\hline$\beta$ & 0.3 \\
$\alpha$ & 0.0001 \\
$\rho$ & 0.025 \\
$\lambda$ & 12.95 \\
$\delta$ & 0.7 \\
$k$ & 700 \\
$K$ & 12000 \\
$\gamma$ & 0.1 \\
$\sigma$ & 1.4 \\
$\phi$ & 3 \\
\hline
\end{tabular}

Under these conditions the internal equilibrium point $P 4$ is a stable focus whose eigenvalues are complex conjugates with negative real part $\left(\varepsilon_{1,2}=-a \pm b i\right)$ as shown in Figure 1(a). This result shows how the temporal evolution of the system which finally falls into a steady state where both population and resources are preserved ${ }^{3}$. Figure $1(\mathrm{~b})$ shows the existence of two basins of attraction, i.e. the system has two steady states that depend on initial conditions. Given an initial condition on $B 1$ the system is sustainable because it approaches to equilibrium $P 4$ and an initial condition on $B 2$ the system is unsustainable because $L, S=0$.

\footnotetext{
${ }^{3}$ For purpose of this paper the term sustainable development is interpreted as any steady-state where $L, S \neq 0$ and can be an equilibrium point, a limit cycle or a chaotic attractor.
} 


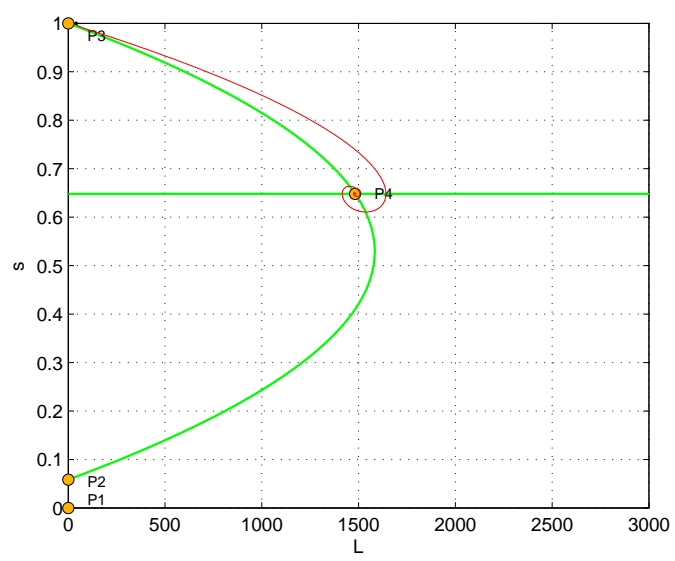

(a)

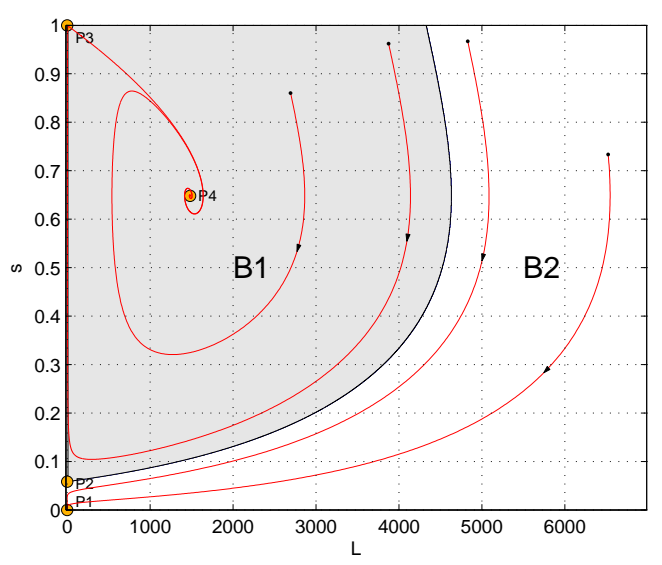

(b)

Figure 1. Phase portrait of system (1): (a) system evolution with nullclines and (b) basins of atraction.

\subsection{Bifurcation analysis}

Previous result shows how by considering constant parameter values, we can understand the system dinamics which is determined by the stability of equilibrium points. Now, the next step is to perform a sensitivity analysis by varying the system parameter values to determine conditions where changes on dynamical behavior occur and system becomes unsustainable. For this, an analysis of bifurcations by using continuation methods reported in [9-10] was developed. Figure 2 shows this result for $\beta$ as the control parameter. As soon as population begins to use resources as a source of income (increasing $\beta$ ) the equilibrium $P 4$ loses stability changing from a stable node to a stable focus to finally becomes an unstable focus through a Hopf bifurcation where the system becomes oscillatory. This bifurcation occurs when an equilibrium has a pair of eigenvalues that cross the imaginary axis creating or destroying a periodic orbit.

\section{A model with two sources of income}

The Ricardo-Malthus model proposed by D'Alessandro [5] consists of two economic activities that exploit two natural resources: forest and land in order to produce wood and corn respectively. The introduction of agricultural as a second source of income is the discussion topic for this section.

\subsection{Mathematical model}

The production in the agricultural sector is assumed to follow the Cobb-Douglas production function $\gamma \lambda(1-\beta)^{\delta} L^{\delta-1}$, where $\lambda$ and $\delta$ are technological parameters in agricultural sector and $\gamma$ is the caloric value of corn. Values of these parameters are also reported in Table 1. The final set of ODEs is system (4). 


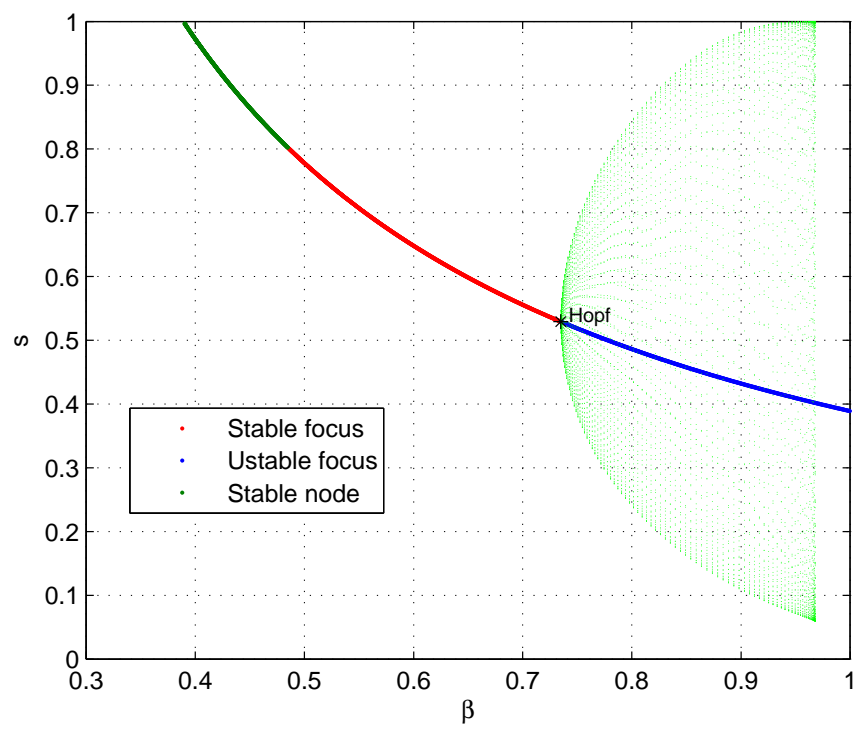

Figure 2. Equilibria continuation and the family of limit cycles asociated to the Hopf bifurcation.

$$
\left\{\begin{array}{l}
\dot{S}=\left[\rho\left(\frac{S}{k}-1\right)\left(1-\frac{S}{K}\right)-\alpha \beta L\right] S \\
\dot{L}=\left(\gamma \lambda(1-\beta)^{\delta} L^{\delta-1}+\phi \alpha \beta S-\sigma\right) L
\end{array}\right.
$$

\subsection{Equilibrium points}

This new system may have four, five, or six equilibria depending on parameter values. A new trivial equilibrium and a new internal equilibrium appear.

Trivial equilibria

$$
\begin{aligned}
& P 1=(0,0), \\
& P 2=(0, k), \\
& P 3=(0, K), \\
& P 4=\left(e^{\frac{\ln \left(\frac{\sigma}{\gamma \lambda}\right)-\delta \ln (1-\beta)}{\delta-1}}, 0\right) .
\end{aligned}
$$

and internal equilibrium $P 5$ and $P 6$ have no analytical solution and must be found numerically through a Newton's method.

\subsection{Steady-state analysis}

The following simulations consider that $30 \%$ of population prefer harvesting resources while $70 \%$ is devoted to agriculture activities, then, $\beta=0.3$. Under these conditions phase portrait is that in Figure 3. In this case the system approaches to a stable limit cycle in the long-run (Figure 3(a)), but depending on initial conditions it can also approach to $P 4$ which 
in this case is a trivial equilibrium where in absence of renewable resources a little population could survive with agriculture as shown in Figure 3(b).

Since analytical solutions for internal equilibria are not available, analytical solutions for eigenvalues are not available too. Then, it is necessary to make it numerically by replacing the numerical values of each equilibrium in the Jacobian matrix. Numerical simulations indicate that if both internal equilibria exist, the lower one $P 5$ is always a saddle while the higher one $P 6$ has variable stability depending on parameter values.

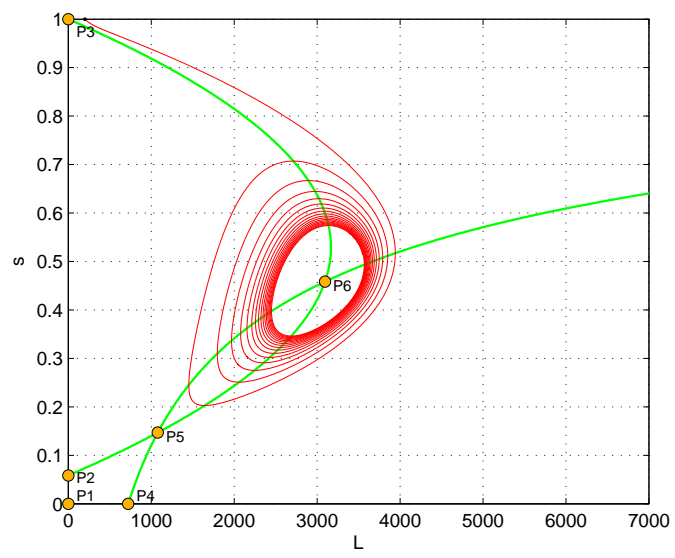

(a)

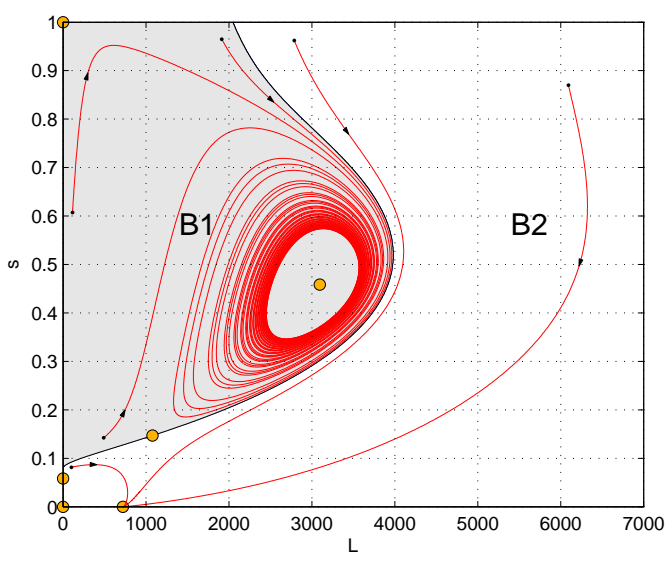

(b)

Figure 3. Phase portrait of system (4): (a) system evolution with nullclines and (b) basins of atraction.

\subsection{Bifurcation analysis}

This part of the work try to explain how non-linear phenomena such as bifurcation help to find regions of sustainability for different sets of parameter values. We found not only several local bifurcations but also global ones, but only those that we consider the most important ones for our purpose are discussed in detail.

To summarize the results we present Figure 4 where both $\beta$ and $\lambda$ are varied in order to find regions of sustainability. For mathematical details about numerical methods for computing bifurcations we recommend to see $[9,10]$. Steady-state for any combination of $\beta$ and $\lambda$ on region $R 1$ is an stable equilibrium, once Hopf bifurcation occur the systems becomes oscillatory and limit cycles appear, this stationary-state occurs on region $R 2$. Homoclinic bifurcation is an important boundary between what is sustainable and what is not because in region $R 3$ there are neither stable equilibria nor stable limit cycles. Finally, $R 4$ is a region where there are no internal equilibria because of the saddle-node bifurcation and any initial condition on this region falls to equilibrium $P 4$. We found two two-parameter bifurcations: the Generalized Hopf $G H$ and the Bodganov-Takes BT which are not interesting in this work. 


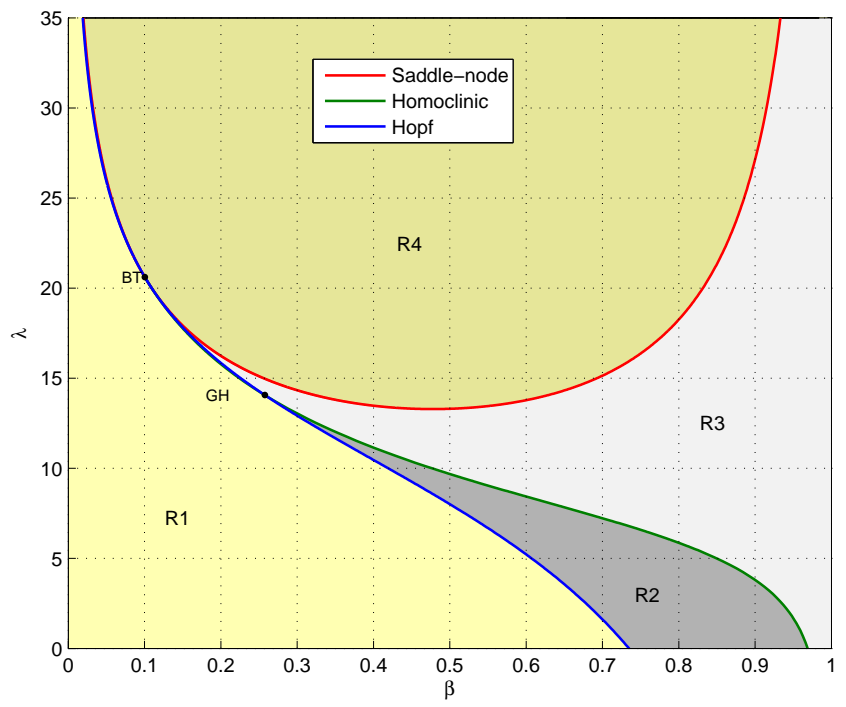

Figure 4. Two-parameter space with regions of sustainability

\section{Technological progress dynamics}

In previous sections long-term dynamic of the interaction between the exploitation of natural resource and population growth was studied. In that models technology used in harvesting $\alpha$ was a system parameter. The aim of this section is to introduce a new differential equation which represents the dynamic behavior of $\alpha$. The proposed ODE introduces the parameter $L_{\min }$ which is the population dedicated to develop this kind of technology, a functional dependence on the existing technology level $\alpha$, and a sort of saturation depending on population level. Our purpose in this section is to present chaotic and quasi-periodic behavior as two new steady-states when modeling sustainable development through differential equations.

\subsection{Technology dynamics}

Equation (6) is the proposed dynamics and parameter values are those in Table 2.

$$
\dot{\alpha}=k_{L} \alpha L^{\delta_{2}}\left[\frac{L-L_{\min }}{L_{\min }^{2}+\left(L-L_{\min }\right)^{2}}\right] .
$$

Table 2. Parameters for technology dynamics.

\begin{tabular}{l|c}
\hline Parameter & Value \\
\hline$\delta_{2}$ & 0.01 \\
$k l$ & 15 \\
\hline
\end{tabular}

\subsection{Steady-state}

In this section we don't use numerical continuation of equilibria to study the steadystate, instead we use Poincare maps which is another useful tool for studying dynamical sys- 
tems. For convenience we select $L_{\min }$ as the Poincare section and detect the crossing of the trajectory in one direction as shown in Figure 5. This result present the existence of a 12periodic orbit in the steady-state. Now it is possible to construct a bifurcation diagram by varying the control parameter $L_{\min }$ (or any other parameter).

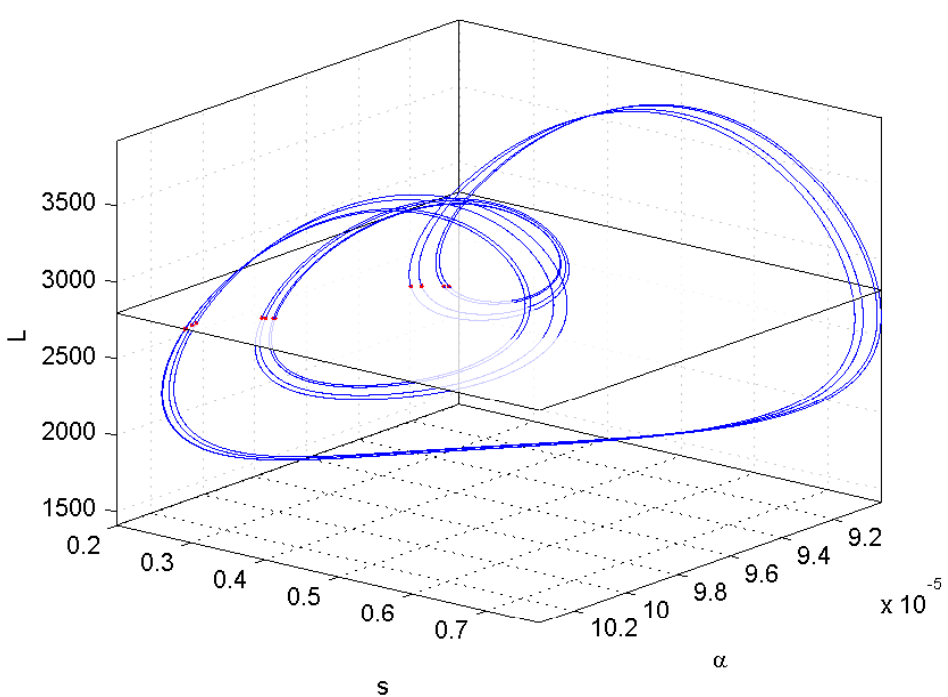

Figure 5. Equilibria continuation and the family of limit cycles asociated to the Hopf bifurcation.

Results in Figure 6 show the possible steady-state scenarios. Non-linear phenomena such as period-doubling bifurcations and chaos in the long run without resources extinction can appear. Region $A$ is characterized by a chaotic attractor or quasi-periodic orbits. In region $B$ the system approaches to stable limit cycles and finally through a Hopf bifurcation located in the boundary between region $B$ and $C$ system can approach to stable equilibria depending on parameter values.

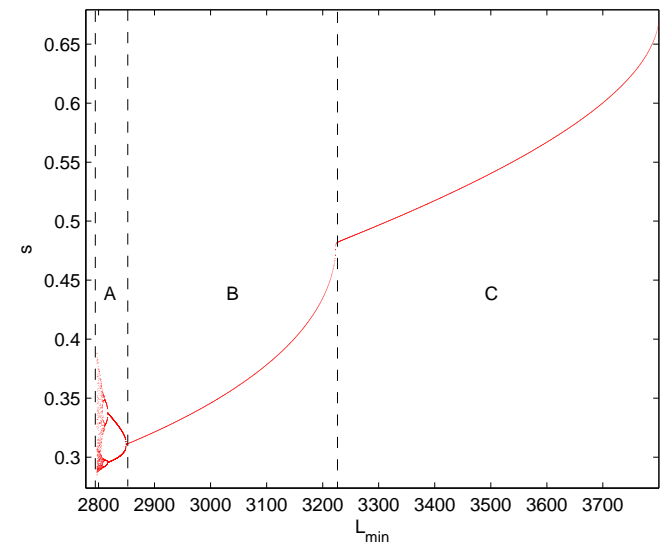

(a)

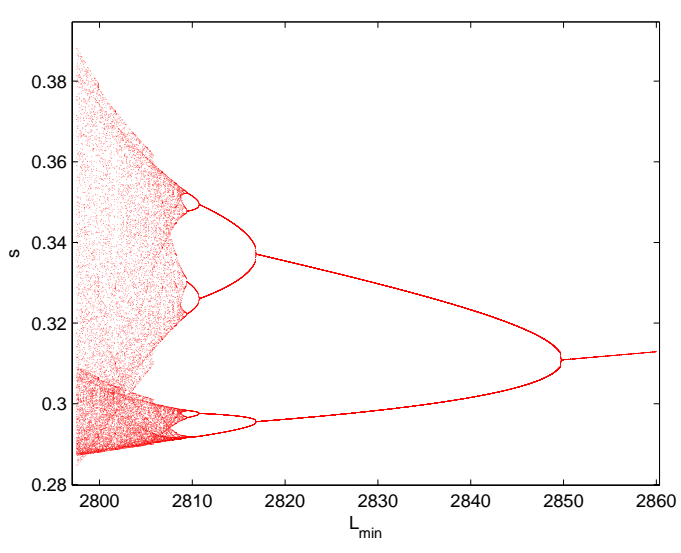

(b)

Figure 6. Bifurcation diagrams 


\section{Mitigation of environmental damage}

This section deals with two ways of mitigating environmental degradation. The first one is related to cultural changes intended to reduce harvesting rates, and the second is related to strict political and institutional changes that seek to preserve an amount or resource.

\subsection{Cultural changes}

So far, parameter $\beta$ has been considered constant an just sensitivity analysis was made in previous sections. Now, we consider that $\beta(s)$ varies depending on the percentage of resources according to the quadratic expression

$$
\beta(s)=\beta_{m} s(3-2 s) .
$$

Equation (7) introduces the fact that economic sector dedicated to harvesting reduce labor when available resources decrease. If the resource level is below $3 / 4$ of their carrying capacity awareness of population changes patterns of consumption. However, in virgin state extraction rate of wood undergoes a temporal increase because of the abundance as shown in Figure 7.

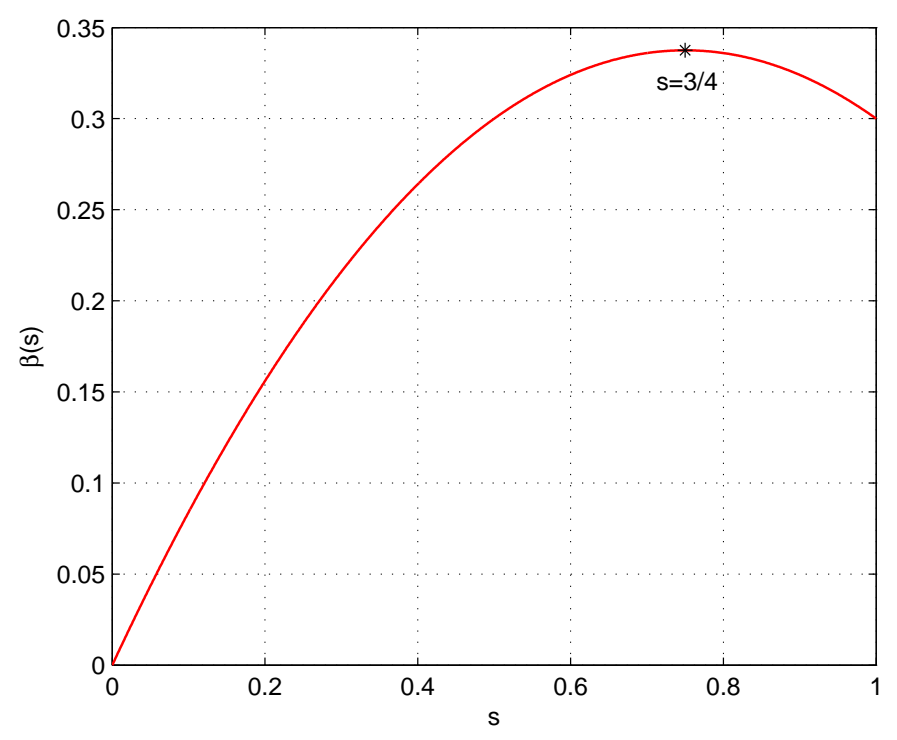

Figure 7. $\beta$ as a function of resources

Figure 8(a) shows stationary state in a constant environment with constant parameters. The introduction of $\beta$ as a function of $s$ change basins of attraction expanding the sustainable basin $B 1$. Namely, it is possible to obtain long-run behaviour with positive population and no resources exhaustion for high initial population level. On the other hand, Figure 8(b) presents continuation of equilibrium points for control parameter $\beta_{m}$ which is the $\beta$ average. In contrast to the results in Section 2 there are no bifurcations and $P 6$ is always stable for any initial condition on $B 1$. This is an interesting result since summarizes the importance of cultural change in the conservation of resources. 


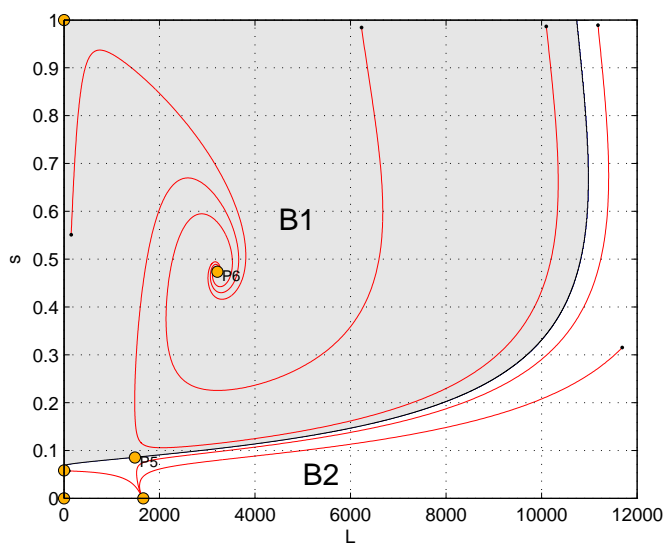

(a)

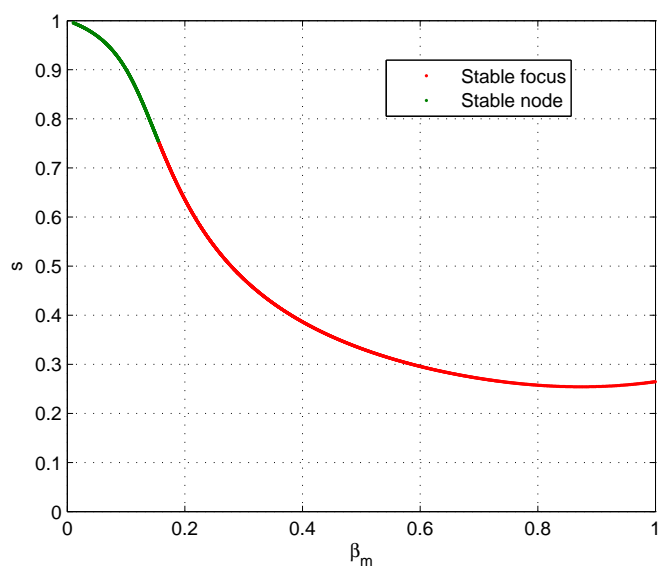

(b)

Figure 8. (a) basins of attraction and (b) continuation of equilibrium points.

\subsection{A piecewise smooth model}

Now, consider a percentage of renewable source $R$ such that below that threshold it is not possible to continue with the excessive harvesting. Once $R$ is crossed, the ecological complex begins to recovery by means of $\beta$ reduction. According to restriction (8) while $s>R, \beta$ will stay at the maximum allowed value $\beta_{\text {max }}$; and if $s<R, \beta$ will reduce in a linear form.

$$
\beta=\left\{\begin{array}{lll}
\beta_{\max } & \text { if } \quad s>R \\
\beta_{\max } s & \text { if } \quad s<R
\end{array}\right.
$$

The second-order Filippov system defined by the single boundary $\Sigma$ and the regions $F_{1}$ and $F_{2}$ is

$$
\begin{aligned}
& \Sigma=\{(L, s): s=R\}, \\
& F_{1}=\{(L, s): s>R\}, \\
& F_{2}=\{(L, s): s<R\},
\end{aligned}
$$

described by the standard ordinary differential equations

$$
\dot{x}=\left\{\begin{array}{l}
f^{1}(x) \text { if } \quad x \in F_{1}, \\
f^{2}(x) \text { if } x \in F_{2},
\end{array}\right.
$$

and the flow that slide according to the Utkin's method is

$$
f^{s}(x)=\frac{f^{1}(x)+f^{2}(x)}{2}+\frac{f^{2}(x)-f^{1}(x)}{2} \theta_{s}(x),
$$

where $\theta_{s}(x)=-\frac{H_{x}\left(f^{1}+f^{2}\right)}{H_{x}\left(f^{2}-f^{1}\right)}$ and $H_{x}$ is the gradient vector.

Finally the sliding region $\Sigma_{s}=\left\{x \in \Sigma:\left|\theta_{s}(x)\right| \leq 1\right\}$ is boundaried by

$$
\begin{aligned}
& T_{1}=\left\{x \in \Sigma: \theta_{s}(x)=-1\right\}, \\
& T_{2}=\left\{x \in \Sigma: \theta_{s}(x)=1\right\} .
\end{aligned}
$$

The obtained sliding limits are: 


$$
\begin{aligned}
& T_{1}=\frac{\rho\left(R K-R^{2}-k K+k R\right)}{\alpha \beta k K}, \\
& T_{2}=\frac{\rho\left(R K-R^{2}-k K+k R\right)}{\alpha \beta R K} .
\end{aligned}
$$

Figure 9(a) shows the phase portrait, sliding region, trajectories for different initial conditions, and the pseudo-equilibrium $P$ when restriction (8) is applied to the planar system (4). Results show that not all initial conditions approaches to positive renewable resources in the long run, nevertheless the established threshold allows an intuitive understanding of institutional change effect on the sustainable development.

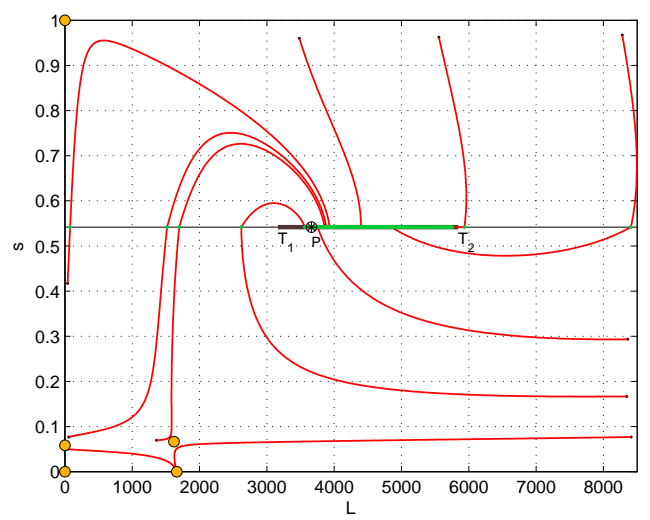

(a)

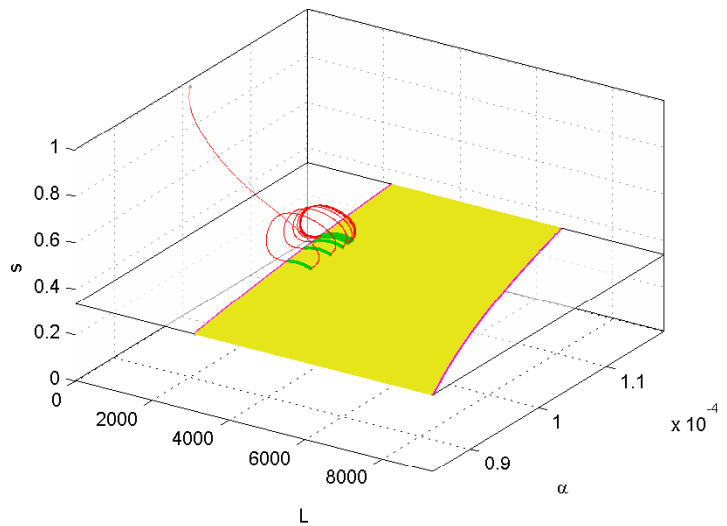

(b)

Figure 9. Filippov systems

Finally, Figure 9(b) presents the evolution of the system when $\alpha$ dynamics is introduced presenting analogous results to the planar system where both the crossing and the sliding region are easy to distinguish.

\section{Final remarks}

The paper studies the dynamic of an endogenous process of population growth in a economy based on resources consumption. The variation in population is assumed to be equal to the difference between calories consumed per-capita and the per-capita natural level of calories needed to survive. This assumption is a representation of a Ricardo-Malthus model where the increasing population is a consequence of the food capacity expansion and only hunger and misery can control it. The introduction of the non-linearity representing agriculture allows the possibility of multiple equilibria and the appearance of local and global bifurcations which help to identify regions of sustainability for different parameter values.

Chaotic and quasi-periodic behavior is an indication of the increasing complexity when new non-linear terms and new differential equations are introduces into a system. These Steady-states are commonly found in many systems and help to identify zones where it is advisable to work, for example in resources management it is convenient to avoid any oscillatory behavior such as limit cycles, chaos, and quasi-periodic orbits characterized by periods of scarcity and abundance. The perfect steady-state would be and stable equilibrium where 
people live in harmony with nature by taking just enough to survive as many indigenous civilizations have done throughout history.

Cultural and Institutional changes were introduced endogenously into the model in order to evaluate at least qualitatively how the dynamics could change and what kind of functions may be introduced so that environmental damage can be mitigated.

\section{Acknowledgement}

We are grateful to $P C I$ and $A B C$ Dynamics groups at Universidad Nacional de Colombia - sede Manizales and the Centro de Excelencia en Investigación Básica y Aplicada (Ceiba) for the financial support.

\section{REFERENCES}

[1] Brander J. A., Taylor M. S., "The Simple Economics of Easter Island: A Ricardo-Malthus Model of Renewable Resource Use". The American Economics Review. 88, 119-138, 1998.

[2] Dalton T. R., Coast R. M., “Could institutional reform have saved Easter Island?”. Journal of Evolutionary Economics. 10, 489-505, 2000.

[3] Reuveny R., Decker C. S., "Easter Island: historical anecdote or warning for the future?". Ecological Economics. 35, 271-271, 2000.

[4] Dalton T. R., Coast R. M., Asrabadi B. R., "Renewable resources, property-rights regimes and endogenous growth". Ecological Economics. 52, 31-41, 2005.

[5] D'Alessandro S., "Non-linear dynamics of population and natural resources: The emergence of different patterns of development". Ecological Economics. 62, 473-481, 2007.

[6] Filippov A. F., "Differential equations with discontinuous right-hand sides". American Mathematical Society Translations. Series 2, AMS, Ann Arbor, 199-231, 1964.

[7] Malthus T. R., "An essay on the theory of population”. Oxford University Press. 1798.

[8] Ricardo D., "Principles of political economy and taxation". (Reprinted) London: Dent, 1817.

[9] Kuznetsov Y. A., "Elements of Applied Bifurcation Theory". Springer-Verlang, Second Edition, New York, Inc., 1998.

[10] Govaerts J. F., "Numerical Methods for Bifurcations of Dynamical Equilibria”. Society for Industrial and Applied Mathematics SIAM, United States of Amarica, 2000.

[11] Angulo F., Olivar G., Osorio G. A., Velásquez L. S., "Nonlinear dynamics and bifurcation analysis in two models of sustainable development". Revista Internacional Sostenibilidad, Tecnologia y Humanismo. 4, 41-46, 2009.

[12] Zhaou, C. M., "Hopf bifurcation in a Ricardo-Malthus model". Applied Mathematics and Computation. 217, 2425-2432, 2010. 